\title{
ESTRATEGIAS SOCIOEDUCATIVAS EN ELÁMBITO FAMILIAR Y COMUNITARIO. UNA ALTERNATIVA DESDE LA CREATIVIDAD SOCIAL PARA LA CONSTRUCCIÓN DE PAZ DESDE LA PRIMERA INFANCIA
}

\author{
Socio-educational strategies in the family and community \\ environment. An alternative from social creativity to build peace from \\ early childhood.
}

\author{
Daniela Arboleda-Cárdenas *; Syndi Johana Toro-Ocampo**
}

Recibido:05 de marzo 2020. Aceptado: 25 de junio de 2020. Publicado: 01 de julio 2020.

Forma de citar este artículo en APA:

Arboleda-Cárdenas, D., Toro-Ocampo, S. (2020, julio-diciembre). Estrategias socioeducativas en el ámbito familiar y comunitario. Una alternativa desde la creatividad social para la construcción de paz desde la primera infancia. Revista CoPaLa, Construyendo Paz. Latinoamericana 10 (año 5), pp. 38-53. DOI:10.35600. 25008870.2020.10.0173, Recuperado desde:

http://revistacopala.net/index.php/ojs/article/view/20

\section{Resumen}

Las estrategias socioeducativas se desarrollan con familias y comunidades para el logro de objetivos referidos al cambio autónomo, mediante la construcción colectiva y la reflexión, para garantizar la atención integral y la garantía de derechos en la primera infancia. Se buscó fundamentar estas estrategias, desarrolladas mediante la propuesta pedagógica de una institución en alianza con actores del territorio, para atender integralmente a poblaciones en condición de vulnerabilidad, desigualdad y exclusión social, a fin de mejorar sus condiciones de vida. La investigación fue posible mediante una metodología dialógico-interactiva que facilitó la interacción con los sujetos, permitiendo dar cuenta de: la relación existente entre las condiciones y características del contexto en el cual se inscriben las estrategias; los elementos constitutivos de las estrategias; la relación entre la fundamentación existente de las estrategias y su aplicación en la realidad práctica; los límites y alcances de las estrategias para el fortalecimiento de la práctica.

\section{Palabras clave}

Estrategias socioeducativas, Ámbito familiar y comunitario, Atención integral, Garantía de derechos.

\footnotetext{
* Trabajadora Social de la Universidad de Antioquia (UdeA); profesional de Buen Comienzo, (Programa de Atención para la Infancia de Antioquia - Colombia), investigadora del Centro de Análisis en Políticas Públicas (CEAPP) de la Facultad de Derecho y Ciencias Políticas (FDCP) de la UdeA; investigadora del Semillero de Estudios en Política Pública (SEPP) adscrito al CEAPP de la FDCP de la UdeA; adscrita al Semillero de Investigación en Familia perteneciente al Grupo de Investigación en Intervención Social (GIIS) del Departamento de Trabajo Social de la UdeA; Medellín (Colombia).daniela.arboledac@udea.edu.co
}

** Trabajadora Social de la Universidad de Antioquia (UdeA); profesional de la Fundación Ximena Rico Llano, Buen Comienzo (Programa de Atención para la Infancia de Antioquia - Colombia), investigadora del Semillero de Investigación en Intervención Social (SIIS) y del Semillero de Investigación en Familia, adscritos al Grupo de Investigación en Intervención Social (GIIS) del Departamento de Trabajo Social de la UdeA; Medellín (Colombia).syndi.toro@udea.edu.co 


\section{Abstract}

Socio-educational strategies are developed with families and communities to achieve objectives relating to autonomous change, through collective construction and reflection, to guarantee comprehensive care and the guarantee of rights in early childhood. These strategies, developed through the educational proposal of an institution in partnership with local actors, are intended to provide comprehensive care for populations in conditions of vulnerability, inequality and social exclusion, in order to improve their living conditions.

The research was made possible by means of a dialogic-interactive methodology that facilitated interaction with the subjects, making it possible to account for: the existing relationship between the conditions and characteristics of the context in which the strategies are inscribed; the constitutive elements of the strategies; the relationship between the existing foundation of the strategies and their application in practical reality; the limits and scope of the strategies for strengthening practice.

\section{Keywords}

Socio-educational strategies, Family and community environment, Comprehensive care, Guarantee of rights 


\section{Planteamiento y justificación de la pregunta de investigación}

La Coordinación de Niñez de Comfenalco Antioquia en alianza con diferentes actores e instituciones hace presencia en varios municipios del departamento por medio de algunos programas destinados a la atención integral de grupos poblacionales en condición de vulnerabilidad, desigualdad y exclusión social, con el fin de mejorar sus condiciones de vida a partir no solo de sus necesidades e intereses, sino también de los saberes y las potencialidades que tienen para aportar significativamente al cambio de sus realidades.

Concretamente, las estrategias socioeducativas en el ámbito familiar y comunitario se desarrollan en tres sectores de Colombia, Medellín (Moravia), Suroeste antioqueño (La Pintada y Bolombolo) y Urabá antioqueño (Apartadó y Chigorodó), mediante las cuales se pretende atender integralmente y garantizar los derechos de los niños y las niñas, por lo cual se generan de manera incluyente y participativa vinculando activamente a familias, actores comunitarios, agentes educativos y actores institucionales. Por consiguiente, la pregunta que dio lugar a esta investigación fue ¿Cuál es la fundamentación en términos epistemológicos, teóricos, metodológicos y deontológicos de las estrategias socioeducativas en el ámbito familiar y comunitario de los Centros de Atención Integral a la Infancia (CAII) de Comfenalco Antioquia?

Así, fundamentar las estrategias socioeducativas dio paso tanto a una justificación argumentada como a la validación científica de las mismas, a partir de criterios que legitiman aquello que se ha dado desde lo experiencial. Sin embargo, la esencia de la fundamentación, más allá de legitimar la práctica socioeducativa que se desarrolla en los CAII mediante las estrategias, fue conocer y ordenar sus lógicas mediante el reconocimiento de la fundamentación existente, la respectiva contrastación con la realidad y las nuevas comprensiones que se generaron a partir de la identificación

de su configuración en términos de componentes y características, las relaciones que tienen entre sí y el porqué de ello, así como de su estructuración y reconstrucción, para lo cual se partió de la experiencia de quienes hacen parte de las estrategias, para comprenderlas, problematizarlas y potenciarlas a partir de su fundamentación.

Adicionalmente, esta fundamentación permitió fortalecer y potenciar algunas prácticas de intervención que apuntan a la transformación de la realidad social, a partir del trabajo con familias y comunidades como agentes 
fundamentales para el desarrollo de dicha intervención; además, es importante mencionar que las estrategias socioeducativas suponen un valor teórico, en tanto fortalecen el conocimiento de cada profesional, pero también para que las familias y comunidades apropien el conocimiento colectivamente construido y, mediante ello, sean conscientes del proceso pedagógico que se lleva a cabo en los CAII, a partir de la permanente reflexión sobre esas otras formas de aprender, desaprender, crear y recrear.

Para la Coordinación de Niñez de Comfenalco Antioquia los resultados derivados de esta investigación permitieron obtener claridades epistemológicas, teóricas, metodológicas y deontológicas, que posibilitaron recuperar y estructurar las estrategias crítica y reflexivamente para que los agentes educativos que hacen parte de la práctica socioeducativa puedan, mediante la reflexión de su experiencia, volver sobre ellas y recrearlas para apropiar, comprender y potenciar su práctica.

En suma, la pertinencia de esta investigación radica en que fue una posibilidad de reconocimiento, reflexión crítica, problematización y nuevas comprensiones sobre la práctica que se lleva a cabo mediante las estrategias, a partir de una lectura problematizadora, compleja y dinámica de sus elementos constitutivos, que a futuro de paso a la acción transformadora ya sea con el fin de fortalecerlas, potenciarlas o generar cambios en estas.

\section{Objetivos}

\section{General}

Fundamentar en términos epistemológicos, teóricos, metodológicos y deontológicos las estrategias socioeducativas en el ámbito familiar y comunitario de los Centros de Atención Integral a la Infancia (CAII) de Comfenalco Antioquia.

\section{Específicos}

Identificar los componentes y las características que configuran las estrategias socioeducativas.

Comprender la relación entre la fundamentación existente de las estrategias socioeducativas y su aplicación en la realidad práctica.

Generar nuevas comprensiones a partir de la identificación de los límites y alcances de las estrategias socioeducativas, para el fortalecimiento de la práctica socioeducativa desarrollada por los agentes educativos.

\section{Referente teórico- conceptual}

El referente teórico que enmarcó esta investigación fue la Educación Social, entendida como un tipo de educación que se desarrolla en ámbitos no formales, promoviendo procesos de formación y sociabilidad integral de los sujetos, desarrollando y potencializando sus capacidades, rescatando las particularidades en el plano individual, familiar, escolar, vecinal y socio-cultural, desde lo cual aporta a la formación social para la 
autonomía, el empoderamiento y la transformación de los sujetos y las comunidades, en el marco del contexto de adversidad, conflicto y crisis social. Principalmente la Educación Social "potencia la transformación cultural, social, económica, política; a partir de ella es posible incidir en los imaginarios colectivos y las prácticas que comprometen conductas preactivas, conductas de riesgo, situaciones de crisis, dificultades circunstanciales" (Arias, 2006, pp. 119-120).

En concreto, la Educación Social busca generar procesos de enseñanza y aprendizaje que le permitan a los sujetos describir, comprender, analizar y transformar su realidad, mediante la identificación de los componentes de la cultura, sus orígenes, y sobre todo la forma en que estos se viven y se representan en la vida social, comprometiendo los escenarios públicos y privados con el fin de promover espacios para la convivencia, fortalecer el tejido social y, mediante esto, generar la transformación, es decir, avanzar en el plano sociopolítico y democrático, en la construcción de paz, el manejo de conflictos, y la superación de la marginalidad y la violencia, por lo cual se promueven prácticas pedagógicas de manera consciente, reflexiva, fundamentada e intencionada, tanto desde el momento de planeación como de implementación y evaluación, mediante la transmisión de valores y la participación activa de los sujetos en la vida social, con el propósito de garantizar los derechos, la democracia y el cambio en la realidad social.

Ahora bien, para la investigación se establecieron unos conceptos sensibilizadores desde el contexto social/situacional y de intervención social que permitieron tener mayores claridades para comprender y orientar el objeto de estudio, los cuales se desarrollan a continuación:

\section{Contexto social/situacional}

Debido a que las dimensiones contextuales de los lugares donde se implementan las estrategias socioeducativas se encuentran transversalizadas por situaciones de vulnerabilidad, desigualdad y exclusión social, fue necesario partir de los siguientes conceptos:

\section{Vulnerabilidad social}

Se puede entender como la dificultad que tiene una comunidad para afrontar un cambio, crisis o situación en específico, lo cual les significa un riesgo en tanto puede generar daño o incertidumbre, dicho riesgo puede ser multifactorial, es decir social, político, cultural, ambiental, etc., además se relaciona con condiciones de un contexto en particular:

[...] se relaciona con los grupos socialmente vulnerables, cuya identificación obedece a diferentes criterios: algún factor contextual que los hace más propensos a enfrentar circunstancias adversas para su inserción social y desarrollo personal, [...] el ejercicio de conductas que entrañan mayor exposición a eventos dañinos, o la presencia de un atributo básico compartido (edad, sexo, condición étnica) que se supone les confiere riesgos o problemas comunes. (Caro, 2003, p. 2) 
En conclusión, la vulnerabilidad social es un fenómeno social estrechamente ligado a la desarticulación social y al resquebrajamiento del tejido social, que a su vez genera condiciones de precariedad y deshumanización, producto de lógicas competitivas e individualistas que ubican a los "menos fuertes", en términos de poder social, político, económico y cultural, en condiciones de riesgo o incluso por fuera del sistema.

\section{Desigualdad social}

Hace referencia al tipo de relaciones sociales que se dan en el marco de una asimetría de poder:

[...] en cuanto atributo de un sistema de relaciones entre conjuntos de seres humanos comprendidos en ellas por su pertenencia al conjunto: la clase social, el grupo étnico, la nacionalidad, el lugar de residencia, sexo, la religión, los hábitos o preferencias de vida, etcétera. No basta que determinados individuos sean - o no sean vistos como- diferentes de otros. La diferencia deviene desigualdad en el momento en que la estructura de poder adjudica determinados efectos a esa diferencia: ejercicio de derechos, acceso a recursos, participación política o social, u otros. Sin esa referencia a las relaciones de poder, a la subjetivación de éstas como ideologías, símbolos y valores y a su objetivación en comportamientos e instituciones, la desigualdad queda reducida a una cuestión de determinados individuos en relación con otros (Vilas, 2007, p. 11).

En conclusión, la desigualdad social puede entenderse como el cúmulo de asimetrías que se presentan en la sociedad por diferentes condiciones pues, como se hizo mención, no responde exclusivamente a un fenómeno social, sino que puede darse a causa de múltiples fenómenos que convergen al mismo tiempo e incluso en una misma población; es por eso que, desde las ciencias sociales, la desigualdad social se ha convertido en un punto de referencia transversal para comprender las brechas que se dan entre diferentes grupos sociales, y que incluso puede ser visto como un sistema que marca límites excluyentes entre diferentes comunidades, afectando el ejercicio democrático.

\section{Exclusión social}

Se refiere a las condiciones de segregación, pauperización, limitación y extrema desigualdad que se dan en el marco de relaciones de poder inequitativas, no por determinación del sujeto excluido, ubicándole por fuera del sistema. Al respecto, Ziccardi (2008) ha desarrollado las dimensiones puntuales a las que responde y que hacen tangible la exclusión social:

Las dimensiones de la exclusión social son, entre otras, las dificultades de acceso al trabajo, al crédito, a los servicios sociales, a la justicia, a la instrucción; el aislamiento, la segregación residencial, la carencia y la mala calidad de las viviendas y de los servicios públicos en los barrios de las clases populares; la discriminación por género a la que están expuestas las mujeres en el trabajo y en la vida social; la discriminación política, institucional o étnico-lingüística que sufren algunos grupos sociales.

La exclusión social hace referencia, entonces, a procesos y prácticas de las sociedades complejas que se convierten en "factores de riesgo social" [...]. (p. 13)

En conclusión, se trata de un fenómeno producto de la lógica de globalización, y es allí donde radica su importancia en tanto permite comprender la realidad social en la actualidad. 


\section{Contexto de intervención desde las ciencias sociales}

Considerando que las estrategias socioeducativas hacen parte de procesos de intervención social que se desarrollan en el ámbito familiar y comunitario, y que a su vez responden a un contexto específico y a una metodología definida e intencionada, es necesario hacer claridad frente a las siguientes categorías ya que permiten ampliar la comprensión frente a esto:

\section{Intervención social}

Según Camelo y Cifuentes (2006), se refiere a un proceso sistemático, lógico, pero sobre todo intencionado al momento de enfrentarse a situaciones problemáticas o necesidades que requieren ser resueltas en una realidad social, mediante acciones que son distinguidas por una ideología y que están orientadas por una clara fundamentación, con el fin de transformar las formas de ver, actuar y sentir de los sujetos que se encuentran inmersos en un contexto específico. En este sentido, la intervención social es un proceso dinámico y de negociación que puede tener múltiples fines, los cuales dependen de las intencionalidades que a su vez se encuentran condicionados por la necesidad sentida de los sujetos, la necesidad creada por la institucionalidad y la necesidad objetiva identificada por el o la profesional que interviene.

\section{Intervención en el ámbito familiar}

Se trata de un proceso de interacción entre el o la profesional y la familia, donde el acompañamiento puede darse ya sea desde la atención y la asistencia a situaciones y necesidades específicas de los sujetos, o desde la orientación de la familia, aunque es necesario aclarar que este tipo de intervención se desarrolla en contextos no clínicos. Concretamente, para la intervención familiar se "requiere una acción experta, la cual puede ser de, facilitación, sostén, control, tutela, mediación o terapia; por medio de la creación conjunta de contextos que expandan las acciones, las cogniciones, los territorios afectivos y nuevas condiciones relacionales" (Quintero, 2006, p. 55).

\section{Intervención en el ámbito comunitario}

Se constituye como un proceso de reflexión producto de estrategias previamente definidas que le apuntan a la solución o tratamiento de la situación que afecta a una determinada comunidad, exenta de supuestos infundados, juicios de valor y mucho menos paternalismos, pues si bien es cierto que gran parte de la intervención social se desarrolla en contextos de vulnerabilidad, desigualdad y exclusión social, en la actualidad los procesos deben trascender del paternalismo que históricamente ha reproducido condiciones de estigmatización, victimización y dependencia, a procesos rigurosos, contextualizados, participativos, dialógicos e incluyentes que promuevan la potenciación y empoderamiento de los sujetos en la búsqueda del cambio social. En este sentido, puede leerse como un sistema de acción local en el cual es necesario identificar 
la base de la comunidad en términos de constitución, relacionamiento, articulaciones, territorialidad, objetivos en común y sentido organizativo, con el fin ya sea de fortalecer sus procesos o de dar respuestas a sus necesidades como comunidad, en virtud del fortalecimiento del tejido social y de las relaciones de solidaridad.

\section{Intervención socioeducativa}

Puede ser entendida como una posibilidad crítica e intencionada de reflexión-acción, que propende por el cambio en la realidad de los sujetos mediante procesos de educación social, donde el rol educador de quien interviene se enfoca en la generación de espacios de concienciación y pensamiento crítico para dicho cambio, pero son los sujetos quienes desde su autonomía y desde sus potencialidades construyen posibilidades para la transformación.

\section{Diseño metodológico}

El desarrollo de la investigación fue posible mediante el trabajo de campo y la práctica socioeducativa que se desarrolla con las familias y comunidades, transversalizado por una estrategia dialógico-interactiva que permitió generar los insumos necesarios que sirvieron como soporte para los resultados del proceso y, con ello, posibilitar el alcance de los objetivos desde una perspectiva relacional circular y solidaria, en la que todas y cada una de las personas participaron del proceso como "investigadores críticos en diálogo” (Freire, 2005, p. 93).

Concretamente, la investigación tuvo cuatro momentos que permitieron desarrollar el proceso a cabalidad y de manera integral: 1) la recolección y generación de la información, para lo cual se desarrollaron varias técnicas, la revisión documental que se dio a partir de textos de carácter académico e institucional, la observación participante que se dio durante todo el proceso, 27 entrevistas semi-estructuradas a diferentes sujetos que hacen parte de la práctica socioeducativa y 3 talleres con las familias de Moravia, La Pintada y Bolombolo; 2) la validación de la información recolectada y generada en campo, con algunos sujetos que hicieron parte del proceso; 3) el análisis e interpretación de la información que se realizó por medio de cuatro operaciones analíticas, la categorización y codificación, la ordenación y clasificación, el establecimiento de relaciones y el establecimiento de redes; 4) la socialización de los hallazgos y resultados investigativos con quienes hicieron parte del proceso.

\section{Análisis de la información}

Para esta investigación se realizaron cuatro operaciones analíticas, la categorización y codificación, la ordenación y clasificación, el establecimiento de relaciones y el establecimiento de redes, mediante herramientas propias para este momento, principalmente matrices de análisis, con el fin de generar los insumos 
necesarios y suficientes para el ejercicio de interpretación y su respectiva concreción, es decir, la fase de escritura del informe final de hallazgos y resultados.

Es necesario aclarar que, aunque el análisis y la interpretación son momentos concretos de lo metodológico, como procesos mentales fueron permanentes en la investigación, es decir, no fueron momentos finales sino transversales, por lo cual durante todo el proceso investigativo se generaron memos analíticos que permitieron plantear algunas alertas en clave de los objetivos.

Por último, conviene aclarar que para este ejercicio se hizo uso de diferentes matrices temáticas que facilitaron el ejercicio de categorización, clasificación y relacionamiento en clave de cinco aspectos referidos a los objetivos de investigación: el reconocimiento y caracterización de los sujetos, la contextualización sociosituacional, los componentes y características de las estrategias socioeducativas, su fundamentación y las nuevas comprensiones sobre dichas estrategias. Adicionalmente, para la operación de establecimiento de redes en torno a las estrategias socioeducativas, se realizó un diagrama analítico de contextos y realidades, un diagrama de sujetos, un diagrama de potencialidades, un mapa descriptivo de ejes estructurantes, un diagrama de componentes y características, algunas tablas de componentes y características por ámbito de intervención, un diagrama de intencionalidades, un diagrama de nuevas comprensiones, un mapa descriptivo de las estrategias y un diagrama analítico sobre las mismas.

\section{Resultados}

En los contextos en los cuales se inscribe la propuesta pedagógica de la Coordinación de Niñez de Comfenalco Antioquia, Moravia, La Pintada, Bolombolo, Apartadó y Chigorodó, se lograron identificar diferentes condiciones problemáticas de vulnerabilidad, desigualdad y exclusión social que inciden considerablemente en la práctica socioeducativa, las cuales se condensan en el siguiente diagrama:

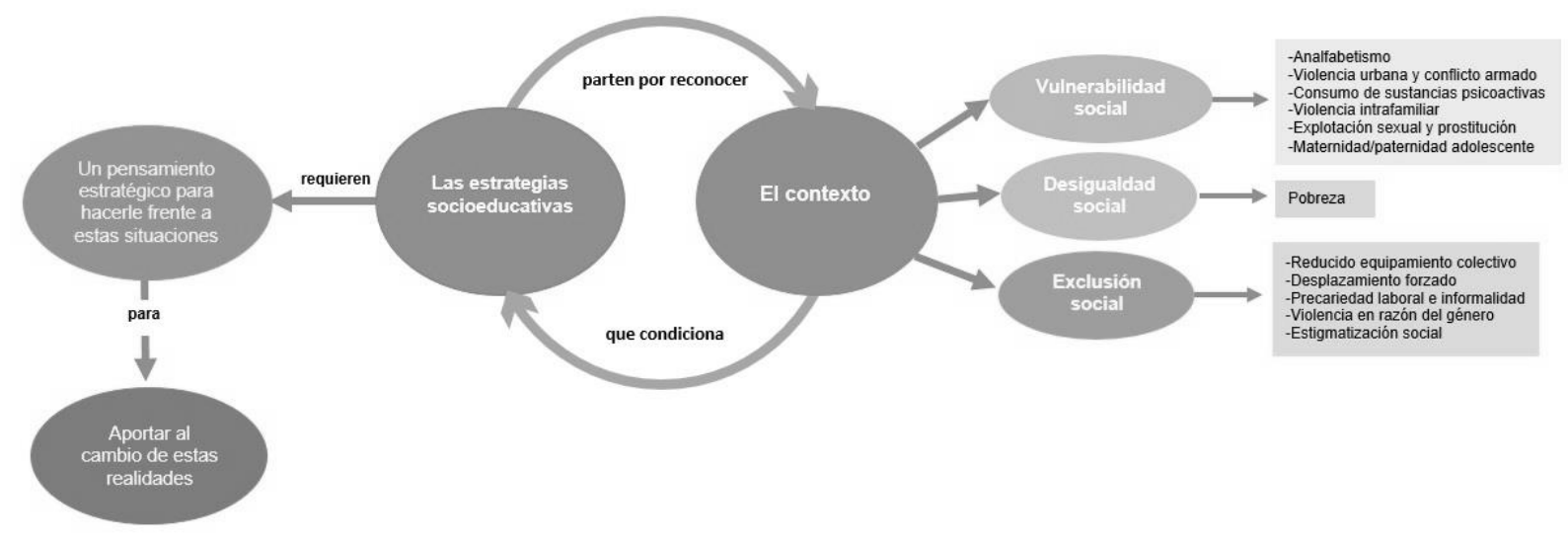

Fuente: elaboración propia, 2017 
En cada uno de estos contextos habitan sujetos que enriquecen la propuesta pedagógica a partir de sus saberes, experiencias y potencialidades; por eso, a continuación, se presenta un diagrama que permite visualizar dichos sujetos, así como sus acciones e intencionalidades:

Mapa de actores

Fuente: elaboración propia, 2017.

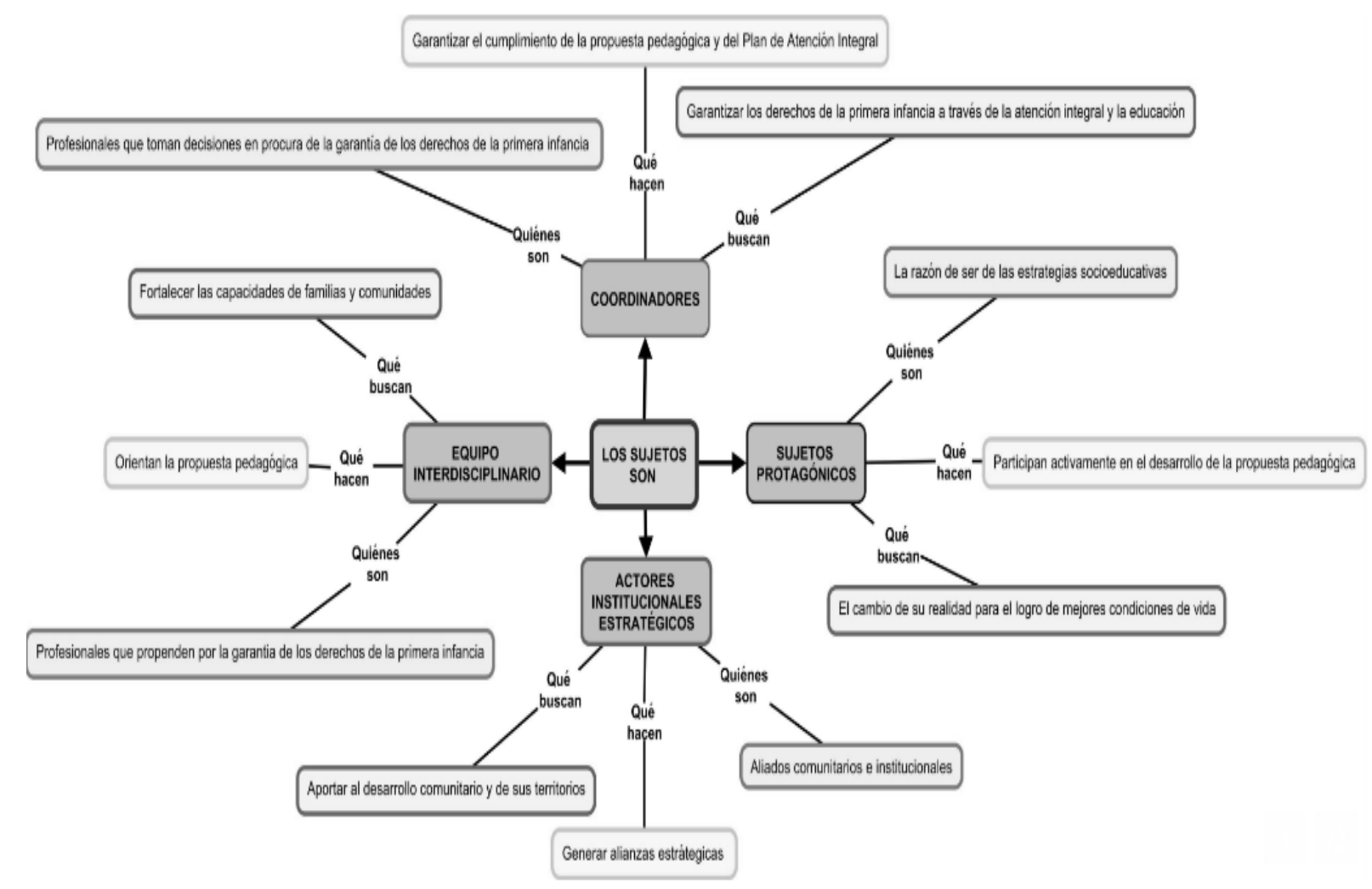

Pese a las condiciones problemáticas de los contextos, un horizonte esperanzador es que en cada territorio convergen múltiples factores potenciales a partir de los cuales se puede hacer frente a estas condiciones para generar alternativas posibles; concretamente, las potencialidades tanto de los contextos como de los sujetos mismos que recrean y dinamizan sus realidades son la oferta institucional, el interés de las familias y comunidades por cualificarse y la interculturalidad de los territorios en los cuales hace presencia la Coordinación de Niñez de Comfenalco

Antioquia. Las estrategias socioeducativas son un conjunto de dispositivos planeados, implementados y evaluados de manera fundamentada, contextualizada e intencionada, de carácter flexible, articulado y reflexivo que, en el marco de procesos de educación social que se llevan a cabo con familias y comunidades, propenden por el logro de objetivos, generalmente referidos al cambio consciente de situaciones que afectan la realidad 
de las personas con quienes se desarrollan, siendo ellas mismas quienes a través del relacionamiento, la interacción, el diálogo, la construcción colectiva y la reflexión propician dicho cambio, lo cual se soporta en dos ejes estructurantes, la atención integral y la garantía de derechos de los niños y las niñas, pero también en unos componentes y características que las configuran, lo cual se ilustra y sintetiza en el siguiente diagrama:

Mapa de ejes estructurantes

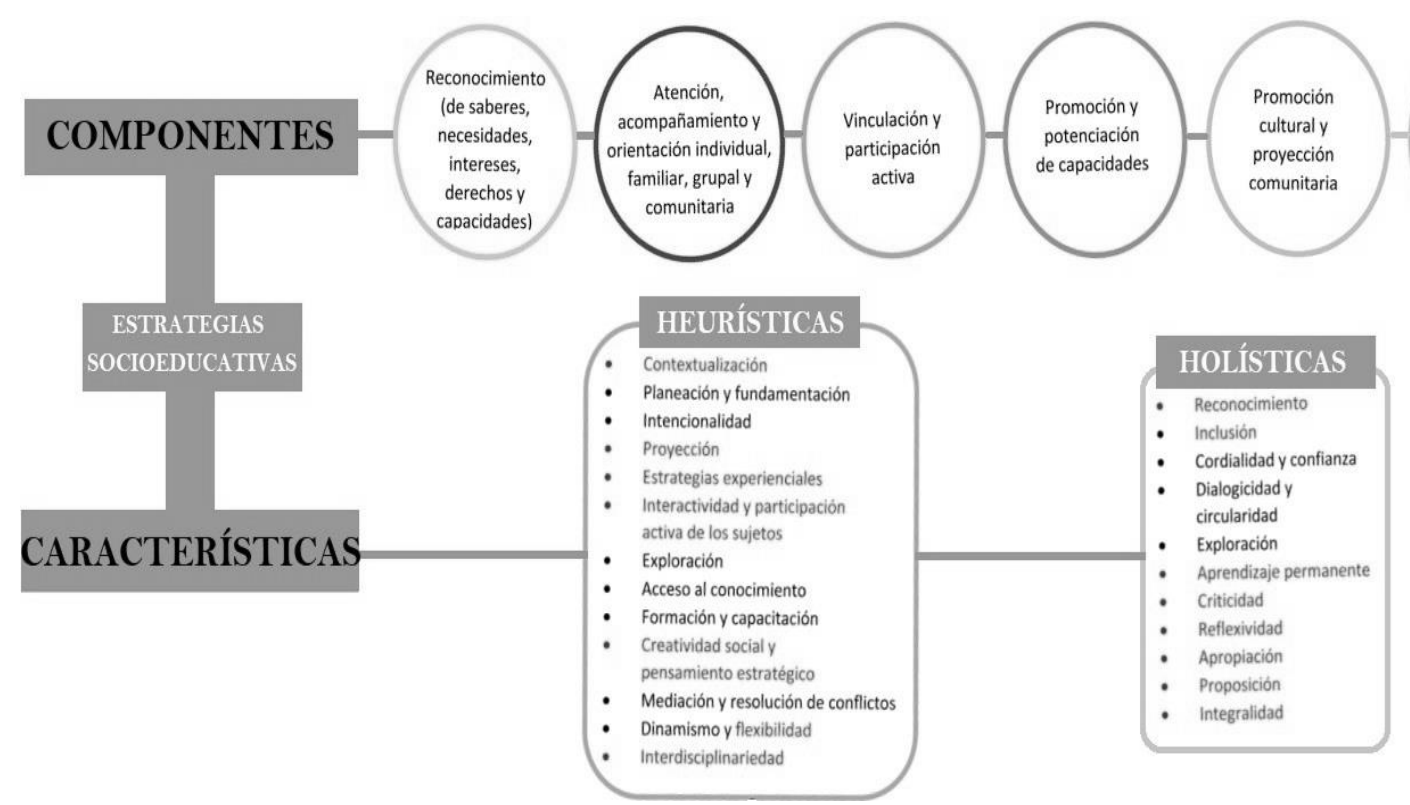

Fuente: elaboración propia, 2017.

Concretamente, en el ámbito familiar se desarrollan siete estrategias socioeducativas: acompañamiento del equipo interdisciplinario, saludos, pasantías, proyectos de investigación, gestación y lactancia materna, acuerdos de convivencia y personaje de la semana; en el ámbito comunitario se llevan a cabo las siguientes:

comunidades protectoras, encuentros de promoción cultural, gestión de proyectos sociales, ludoteca experimental y llegaron las cartas; pero también hay algunas estrategias que se desarrollan en ambos ámbitos, la comunidad de diálogo y los encuentros formativos en temas de interés.

Conviene anotar que dichas estrategias se enmarcan en unas intencionalidades que permiten poner en práctica la propuesta pedagógica y facilitan el logro de los objetivos propuestos, pero no de manera aislada, sino que se relacionan y complementan entre sí, y son las siguientes: 
Diagrama intencionalidades

Fuente: elaboración propia, 2017.

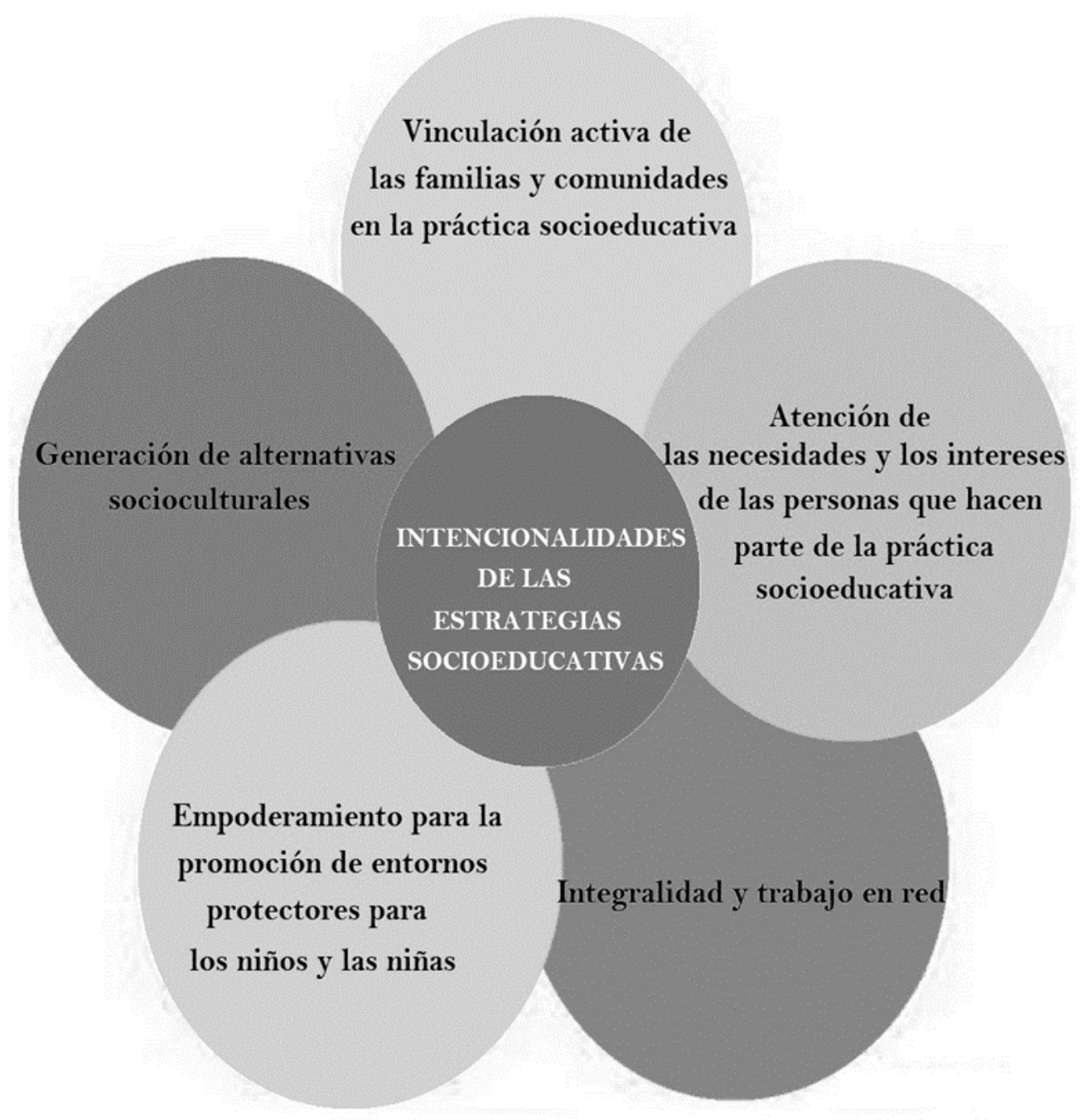

En este sentido, se generaron nuevas comprensiones a partir de la exploración de los límites y alcances de las estrategias socioeducativas, lo cual permite aportar al fortalecimiento de la práctica socioeducativa; por eso, a continuación, se presenta un diagrama que facilita la comprensión de las estrategias en términos tanto de los retos y desafíos que aún tiene la práctica, así como de los asuntos que la potencian: 


\section{Diagrama nuevas comprensiones}

Fuente: elaboración propia, 2017.

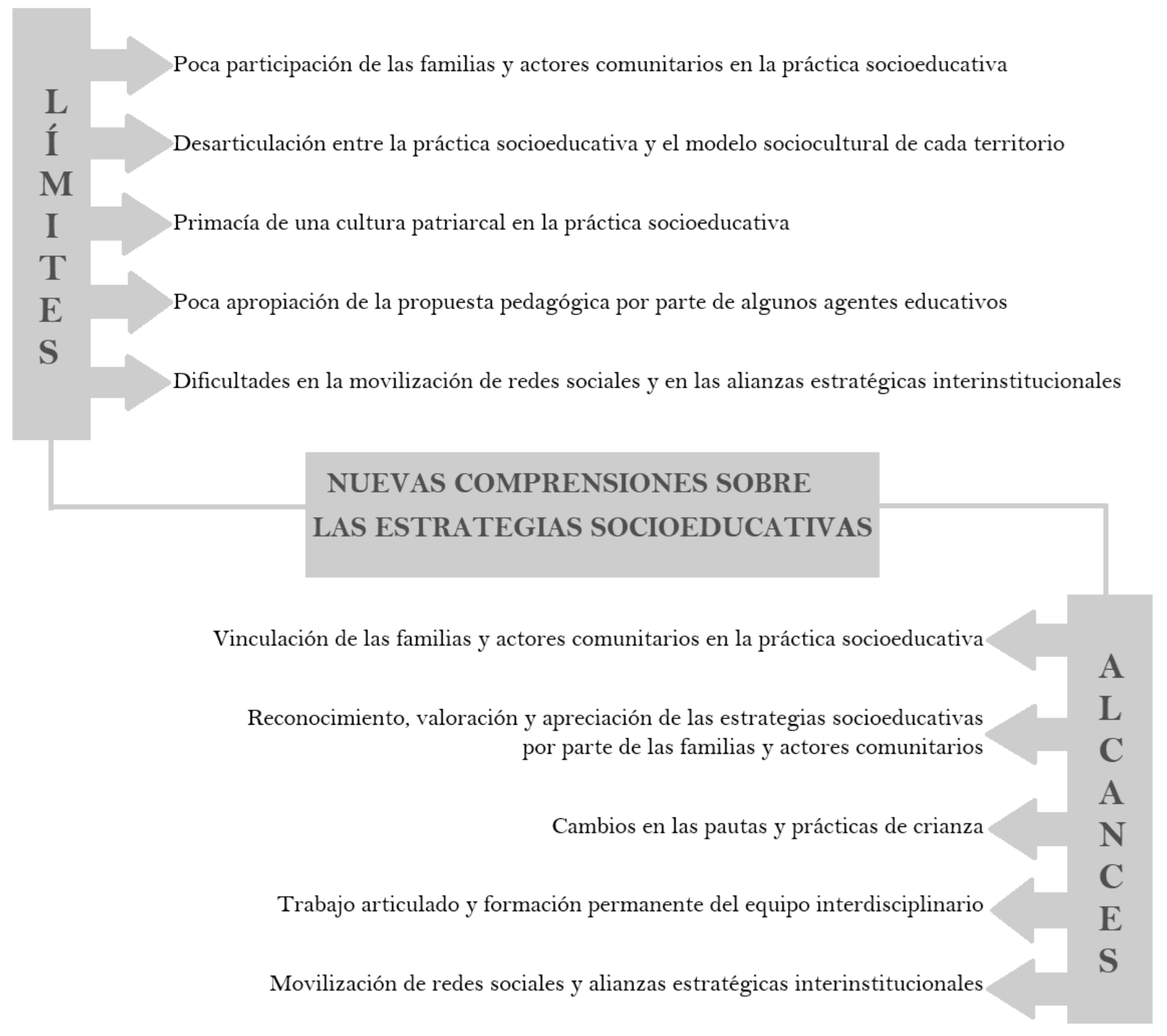

Para esta investigación la fundamentación, en tanto eje estructurante, fue entendida como un proceso sistemático, crítico y holístico de reflexión y comprensión que permite develar los elementos que constituyen la esencia de las prácticas y, mediante ello, poder dar sustento a las mismas a partir de la experiencia de los sujetos que hacen parte de estas, posibilitando que estos sujetos puedan apropiarse y dar cuenta de aquello que soporta su quehacer profesional para evitar prácticas espontáneas e irreflexivas que no aportan al cambio de realidades.

Por lo tanto, se desarrolló crítica, reflexiva, analítica y propositivamente una fundamentación en términos epistemológicos, teóricos, metodológicos y deontológicos, a partir de un relacionamiento entre estos fundamentos y su aplicación en la realidad práctica, reconociendo que se trata de una práctica socioeducativa 
integral por lo que ninguno de estos fundamentos se da de manera aislada, sino que son interdependientes, dialogantes y se complementan entre sí; así, con el fin de develar esa conexión e interdependencia que existe entre los diferentes fundamentos, a continuación se introduce un diagrama analítico que permite desentrañar, reconocer y reconstruir los fundamentos propios que constituyen dicha práctica:

\section{Conclusiones y recomendaciones}

En el plano epistemológico se encontró que gran parte del equipo interdisciplinario de los diferentes CAII conciben y nombran su objeto de intervención, es decir, las estrategias socioeducativas, como un proceso que debe darse de manera: contextual, en tanto se parte de las características y necesidades de los niños, niñas, familias y actores comunitarios; intencional, ya que son orientadas por unos objetivos con los cuales se pretende responder a dichas necesidades; e integral, porque se cuenta con el acompañamiento de un equipo interdisciplinario para el desarrollo del proceso, en clave de la atención integral y la garantía de derechos de los niños y las niñas que hacen parte de los CAII.

En lo que respecta a los referentes teóricos que sirven para dar fundamento a las estrategias, hay que resaltar que los lenguajes del arte son la expresión más presente dentro del discurso del equipo interdisciplinario de cada CAII, pues reconocen y enuncian que esta es una posibilidad de que todas las personas puedan participar, ya que el arte es un lenguaje universal, pero que a su vez es la posibilidad de transmitir y construir conocimiento a partir de diferentes expresiones, sentires, saberes, entre otros.

También en el orden teórico se encontró que en términos de la relación educación-cultura, la generalidad se encuentra en una relación de ida y vuelta, en el sentido de que la práctica socioeducativa se encuentra permeada por todo el acumulado cultural de los niños, niñas, familias y actores comunitarios, lo cual en ocasiones puede incidir negativamente sobre el desarrollo de la misma; sin embargo, también es recurrente en lo expresado por gran parte del equipo que con las estrategias socioeducativas se logran cambios significativos en términos socioculturales. No obstante, los planteamientos teóricos de tipo socio-crítico están menos arraigados en el discurso o, por lo menos, es algo poco explícito en relación a los lenguajes del arte y al indagar por este aspecto el equipo hace más referencia al desarrollo del pensamiento crítico en los sujetos con quienes se desarrollan las estrategias socioeducativas, que a asumir una postura crítica como profesionales frente a la realidad en la cual se desarrollan dichas estrategias.

En términos metodológicos, la mayoría de profesionales que participaron del proceso investigativo y quienes día a día desarrollan su quehacer en virtud de las estrategias, plantearon que el fundamento de la metodología de dichas estrategias son las necesidades y los intereses de las personas, es decir, de los niños, niñas, familias 
y actores comunitarios que son la razón de ser de las mismas, también expusieron que los impactos o resultados esperados del proceso en el ámbito familiar y comunitario se dan en el largo plazo; por su parte, la generalidad evidenciada en las expresiones y narrativas de las familias es que es una metodología vinculante, pues constantemente permite su participación en la práctica socioeducativa.

Al indagar por los principios y apuestas que orientan las estrategias, sobresalen asuntos como: tener presente y respetar los intereses de los sujetos; generar procesos socioeducativos que posibiliten transformaciones en el ámbito familiar y comunitario; muy relacionado con el paradigma que guía la propuesta pedagógica, es decir el socio-crítico, propiciar en los sujetos capacidad de autonomía y criticidad en torno a su realidad para que, en esa medida, puedan cuestionarse, reflexionar y proponer sobre las dinámicas que el día a día les pone en frente.

En términos generales, algo transversal y sumamente significativo es que tanto el equipo interdisciplinario como las familias y los agentes comunitarios enuncian su experiencia anclada a la atención integral y a la garantía de derechos de los niños y las niñas.

En cuanto al desarrollo de las estrategias socioeducativas, es de suma importancia seguir apostándole a metodologías experienciales y participativas que permitan un aprendizaje más significativo de los mensajes y contenidos que se abordan con las familias y las comunidades.

Durante el desarrollo del proceso investigativo se pudo evidenciar la importancia de reflexionar y fundamentar la práctica socioeducativa, ya que es la posibilidad fehaciente de problematizar y, a partir de allí, potenciar el proceso y posicionar otras formas de ver, comprender y hacer; en otras palabras, es una fuente de aprendizaje significativo y transformador en tanto incita nuevas búsquedas, horizontes, sentidos y significados desde y para la práctica, pues lo que se encamina al cambio de las prácticas es lo que proporciona sentido al hacer, es decir, reconocer, problematizar, comprender y poder dar razón del quehacer es precisamente donde cobra sentido la acción, en tanto permite generar nuevas comprensiones en términos de límites y alcances frente a la práctica. En este sentido, es necesario que los agentes educativos sigan abriendo espacio a la reflexión crítica sobre su práctica desde la "curiosidad epistemológica" a la que invita Freire (1997), aquella que permite desentrañar y develar crítica y reflexivamente asuntos contenidos, y quizá ocultos, en la práctica y en la realidad en la cual se inscribe esta; así mismo, es fundamental que quienes no lo han incorporado a su quehacer reconozcan la importancia de ello para garantizar "prácticas generadoras de saber" (Ghiso, 2006) y, por tanto, de transformación en permanente diálogo con la propuesta pedagógica; adicionalmente, es importante reconocer, problematizar y reflexionar la práctica socioeducativa pero no de manera aislada, sino en el marco del contexto 
histórico, social, político y cultural en el cual se inscribe, pues esta se ve condicionada en gran medida por factores y situaciones propias de cada territorio que inciden potencialmente en los cambios o permanencias que se generan sobre todo en el modelo sociocultural.

\section{Referencias bibliográficas}

Arias, R. (2006). Educación Social y formación ciudadana en tiempos de globalización. En: Revista Tendencia \& Retos (No. 11). Bogotá: Universidad de La Salle. Páginas: 117-129.

Camelo, A. y Cifuentes, R. (2006). Aportes para la fundamentación de la intervención profesional en Trabajo Social. En: Revista Tendencia \& Retos (No. 11). Bogotá: Universidad de La Salle. Páginas: 169-187.

Caro, E. (2003). LA VULNERABILIDAD SOCIAL COMO ENFOQUE DE ANÁLISIS DE LA POLÍTICA DE ASISTENCIA SOCIAL PARA LA POBLACIÓN ADULTA MAYOR EN MÉXICO. Santiago de Chile: CEPAL.

Freire, P. (1997). Política y educación. México: Siglo Veintiuno Editores.

Freire, P. (2005). Pedagogía del oprimido. México: Siglo Veintiuno Editores.

Ghiso, A. (2006). Prácticas generadoras de saber. Reflexiones freirianas en torno a las claves de la sistematización. Recuperado de http://virtual.funlam.edu.co/repositorio/sites/default/files/repositorioarchivos/2011/05/practicas_generadoras_ saber_ponencia_aghiso_recurso_propio_unidad_3.895.pdf

Quintero, A. (2006). DICCIONARIO ESPECIALIZADO EN FAMILIA Y GÉNERO. En: Revista Interamericana de Bibliotecología (Vol. 29 No. 02). Medellín: Universidad de Antioquia. Páginas: 61-78.

Vilas, C. (2007). Desigualdad social y procesos políticos: una perspectiva interdisciplinaria. Mendoza: Universidad Nacional de Cuyo.

Ziccardi, A. (2008). Procesos de urbanización de la pobreza y nuevas formas de exclusión social. Los retos de las políticas sociales de las ciudades latinoamericanas del siglo XXI. Bogotá: Siglo del Hombre Editores. 